\title{
DECONVOLUTION OF SEISMIC DATA USING PHASE CHANGE OPERATORS
}

\author{
Dorian Caraballo Ledesma and Milton José Porsani
}

\begin{abstract}
This paper presents a new approach for wavelet deconvolution. We investigate the use of all-pass operator to change the phase of the minimum-phase inverse filters. The all-pass operators are formed by using the roots of the polynomial associated to the Wiener-Levinson filters. By selecting subsets of roots it is possible to generate an ensemble of mixed-phase inverse filters. The optimization process to obtain an optimum inverse filter is performed by using a genetic algorithm. We have used the varimax norm as the objective function to measure the simplicity of the seismic trace after deconvolution. The method was tested using synthetic and real seismic data.
\end{abstract}

Keywords: mixed-phase deconvolution, seismic data processing, wavelet estimation.

RESUMO. Apresentamos uma nova abordagem para deconvolução do pulso sísmico. Investigamos o uso de operadores de mudança de fase para obtenção de filtros de fase mista a partir de filtros de fase mínima. Os operadores de mudança de fase são construídos a partir das raízes associadas aos filtros de Wiener-Levinson. Escolhendo subconjuntos daquelas raízes é possível gerar um conjunto de filtros inversos de fase mista. A escolha do filtro ótimo é realizada através do método de otimização conhecido com algoritmo genético. Para tanto utilizamos a norma varimax para medida da simplicidade do traço deconvolvido. 0 método foi testado utilizando dados sísmicos sintéticos e reais.

Palavras-chave: deconvolução de fase mista, processamento sísmico, estimativa do pulso sísmico. 


\section{INTRODUCTION}

Among geophysical methods, the seismic reflection method is the most used in the exploration of hydrocarbons. It allows, like no other, obtaining an image of the subsurface that clearly reflects the geology of the sedimentary basins. Improving the quality of seismic images and increasing the ability to identify and discriminate the seismic reflectors is one of the biggest challenges of the seismic reflection method. Aiming to improve the resolution, quality and fidelity of seismic imaging, new methods of processing and filtering seismic data are being continuously developed.

The deconvolution filter is a signal treating procedure that has important applications in various scientific fields such as radar signal and astronomical image processing, in order to improve the sharpness of celestial images. In seismic oil exploration, particularly in the study of reservoirs, deconvolution is an important step of seismic processing, applied to improve temporal resolution of traces, allowing better top and bottom identification of thinner layers and thus better definition of subsurface geology. When used for this purpose, it is called wavelet or spiking deconvolution (Robinson, 1967; Robinson \& Treitel, 1982). Deconvolution is also used to attenuate multiple reflections that occur when the seismic energy is reflected more than once at each interface. In this case, it is called predictive deconvolution and the multiple reflections are considered as noise to be eliminated (Robinson \& Treitel, 1982).

Wavelet deconvolution aims to compress wavelet shape in order to recover reflectivity function or impulse response of the medium. In most cases, the wavelet is not known and is available only as an estimate of its autocorrelation function, which is obtained directly from the seismic traces. The classical method known as the Wiener-Levinson deconvolution (WL) does not provide the best results when the wavelet is not minimum phase. The WL method limitations are well known and several authors have investigated methods and strategies that would provide good results for realistic oil exploration situations, when the wavelet is not necessarily minimum phase or when the reflectivity is not necessarily random.

In the last two decades, several papers on deconvolution and wavelet estimation have been published in Geophysics literature. Most often the authors focus on and try to solve problems related to the wavelet phase character (Levi \& Oldenburg, 1982; Eisner \& Hampson, 1990; Ulrych \& Treitel, 1991; Lazear, 1993; Leinbach, 1993; Velis \& Ulrych, 1996; Porsani \& Ursin, 1998, Porsani \& Ursin, 2000; Ursin \& Porsani, 2000, Sacchi \& Ulrych, 2000; Misra \& Sacchi, 2006; Lu \& Wang, 2007; Van, 2008; Van \& Pham, 2008; Misra \& Chopra, 2010).
Porsani \& Ursin (1998) used the solution of Yule-Walker extended equations and proved that it is possible to obtain mixedphase filters associated with minimum phase wavelet. These authors estimated the polynomial roots associated with the minimum phase wavelet and, then obtained mixed-phase filters using all-pass operators. Thus, a large number of mixed-phase filters can be obtained and used in wavelet deconvolution. The optimal filter is then obtained by solving the optimization problem via genetic algorithm (GA), Holland (1975) and Goldberg (1989).

In this paper, a new procedure for simplifying the deconvolution method originally proposed by Porsani \& Ursin (1998) is presented. This new approach is tested and validated using synthetic and real seismic data.

\section{BASIC CONCEPTS}

\section{Seismic trace convolution model}

In the linear system theory, the seismic trace can be represented as the result of the wavelet convolution with the impulse response of the medium, also called reflectivity function Yilmaz (1987) Mathematically expressed as,

$$
x_{t}=p_{t} * r_{t}+\eta_{t},
$$

where:

$x_{t}=$ seismic trace;

$p_{t}=$ wavelet of every reflected event;

$r_{t}=$ impulsive response or reflectivity function;

$\eta_{t}=$ additive noise.

Figure 1 shows the seismic trace convolution model.

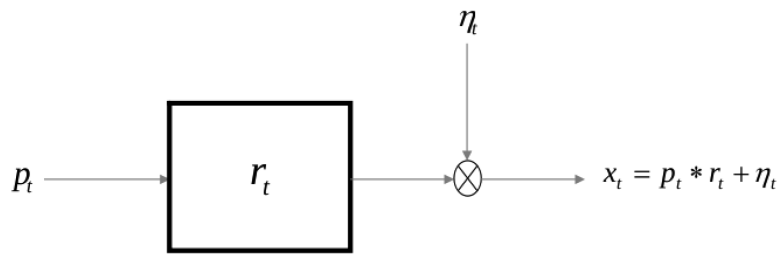

Figure 1 - Linear system model representing the seismic trace.

The reflectivity function can be retrieved using the seismic trace convolution model, where $c_{t}$ is the inverse wavelet filter $\left(c_{t}=p_{t}^{-1}\right)$, as shown below.

$$
c_{t} * p_{t}=\delta_{t},
$$

where: $\delta_{t}$ is Kronecker delta. Convolving $c_{t}$ with equation (1), we 
obtain:

$$
\begin{aligned}
\tilde{e}_{t}=c_{t} * x_{t} & =c_{t} * p_{t} * e_{t}+c_{t} * v_{t} \\
& =\delta_{t} * e_{t}+c_{t} * v_{t} \\
& =e_{t}+c_{t} * v_{t}
\end{aligned}
$$

Considering negligible the noise $\eta_{t}$, we verify that the convolution of the trace with the inverse filter restores the reflectivity function. In this case, we say that the seismic trace was deconvolved.

Deconvolution of the wavelet is, therefore, the convolution of the wavelet with its inverse. The inverse filter when convolved with a seismogram extracts the impulse response from the medium, that is, it retrieves the reflectivity function.

\section{Wiener-Levinson deconvolution}

The conventional Wiener-Levinson method used to deconvolve a wavelet is also known as minimum phase deconvolution. The polynomials $C(Z)$ whose roots in the complex $(Z)$ plane are outside the unit radius circle are called minimum phase. When the shape of the wavelet is not known (most common case), the inverse filter is calculated using the statistical method of least squares (Yilmaz, 1987). This method has three basic steps:

(i) estimating the coefficients of the autocorrelation function (ACF),

(ii) obtaining the Wiener-Levinson (WL) inverse filter, and

(iii) applying the filter(s) to the seismic(s) trace(s) by convolution $\left(c_{t} * x_{t}\right)$ (deconvolution).

In the WL method, the inverse filter is obtained by solving the normal equations. Such filters are causal $c_{t}, t=0, \ldots, N$, and minimum phase (Ulrych \& Treitel, 1991) so that they can be the inverse of minimum phase wavelet since only these have causal inverse (Robinson, 1967). Consequently, the WL method basic assumption is that the wavelet of the seismic(s) trace(s) is minimum phase.

Another limitation of WL method is associated with the FAC used to obtain the inverse filter. Considering that the wavelet is not available, its autocorrelation is estimated directly from the seismic(s) trace(s) that needs to be deconvolved. In this case trace FAC coefficients, $R x x(t)$, represent a good estimate of the FAC wavelet, $R p p(t)$, only when the reflectivity function, $e(t)$ is represented by a random series that does not contribute (or interferes) with the inverse filter. Only then, at least theoretically, the FAC estimated directly from the seismic traces is a good estimate of the FAC wavelet (Robinson \& Treitel, 1982; Yilmaz, 1987; Leinbach, 1993; Porsani \& Ursin, 1998).

\section{Wiener-Levinson filter}

The WL filter is usually obtained by the least square method. Using the linear predictive model to represent the seismic trace $x_{t}$, we can write:

$$
\tilde{x}_{t}=\tilde{a}_{1} x_{t-1}+\cdots+\tilde{a}_{N} x_{t-N}
$$

where the prediction error (or residue) is $e_{t}=x_{t}-\tilde{x}_{t}$ and also given by:

$$
e_{t}=x_{t}-\tilde{a}_{1} x_{t-1}+\cdots+\tilde{a}_{N} x_{t-N}
$$

This equation can be represented in matrix form:

$$
e_{t}=\left[\begin{array}{llll}
x_{t} & x_{t-1} & \cdots & x_{t-N}
\end{array}\right]\left[\begin{array}{c}
1 \\
\tilde{c}_{1} \\
\vdots \\
\tilde{c}_{N}
\end{array}\right]
$$

when $\tilde{c}_{j}=-\tilde{a}_{j}$, the matrix for a filter with $N=3$ coefficients is:

$$
\left[\begin{array}{c}
e_{0} \\
e_{1} \\
e_{2} \\
e_{3} \\
\vdots \\
e_{M} \\
e_{M+1} \\
e_{M+2} \\
e_{M+3}
\end{array}\right]=\left[\begin{array}{cccc}
x_{0} & 0 & 0 & 0 \\
x_{1} & x_{0} & 0 & 0 \\
x_{2} & x_{1} & x_{0} & 0 \\
x_{3} & x_{2} & x_{1} & x_{0} \\
\vdots & \vdots & \vdots & \vdots \\
x_{M} & x_{M-1} & x_{M-2} & x_{M-3} \\
0 & x_{M} & x_{M-1} & x_{M-2} \\
0 & 0 & x_{M} & x_{M-1} \\
0 & 0 & 0 & x_{M}
\end{array}\right]\left[\begin{array}{c}
1 \\
\tilde{c}_{1} \\
\tilde{c}_{2} \\
\tilde{c}_{3}
\end{array}\right] .
$$

The quadratic form obtained by the summation of the squared residuals $\sum_{i} e_{i}^{2}=Q(\tilde{c})$ can be minimized with respect to $\tilde{c}_{j}, j=1, \ldots, N$ parameters, resulting in a normal equation system (NEs) shown below as an enlarged matrix,

$$
\left[\begin{array}{cccc}
r_{0} & r_{-1} & \ldots & r_{-N} \\
r_{1} & r_{0} & \ddots & \vdots \\
\vdots & \ddots & \ddots & r_{-1} \\
r_{N} & \ldots & r_{1} & r_{0}
\end{array}\right]\left[\begin{array}{c}
1 \\
\tilde{c}_{1} \\
\vdots \\
\tilde{c}_{N}
\end{array}\right]=\left[\begin{array}{c}
E_{\tilde{c}, N} \\
0 \\
\vdots \\
0
\end{array}\right]
$$

where $E_{\tilde{c}, n}$ is the total sum of minimized squared residuals and $r_{k}$ is the coefficient $k$ of the seismic trace FAC.

$$
r_{k}=\sum x_{t} x_{t+k} .
$$

The WL method yields good results based on the following assumptions: (i) the wavelet is minimum phase, (ii) the sig$\mathrm{nal} / \mathrm{noise}$ ratio is high and, (iii) the reflectivity function is random. 


\section{The Wiener-Levinson minimum phase wavelet}

Once the WL filter is causal (see Eq. (7)) and minimum phase (Ulrych \& Treitel, 1991), the corresponding inverse polynomial $\tilde{P}(Z)=1 / \tilde{C}(Z)$ is also minimum phase and causal. Consequently, the minimum phase wavelet can be obtained directly and in a numerically stable way by the method of polynomial division. This is a simple method where the minimum-phase wavelet coefficients $\left\{\tilde{p}_{j}\right\}$ are calculated by retro-substitution, from the smallest to largest indices, since the system is lower triangular, as shown below.

$$
\left[\begin{array}{ccccc}
1 & 0 & \ldots & \ldots & 0 \\
\tilde{c}_{1} & 1 & 0 & \ddots & \vdots \\
\tilde{c}_{2} & \tilde{c}_{1} & 1 & \ddots & \vdots \\
\vdots & \vdots & \vdots & \ddots & 0 \\
0 & \tilde{c}_{N} & \ldots & \tilde{c}_{1} & 1
\end{array}\right]\left[\begin{array}{c}
1 \\
\tilde{p}_{1} \\
\tilde{p}_{2} \\
\vdots \\
\vdots \\
\tilde{p}_{N}
\end{array}\right]=\left[\begin{array}{c}
1 \\
0 \\
\vdots \\
\vdots \\
\vdots \\
0
\end{array}\right] .
$$

\section{Varimax Norm}

The varimax norm was introduced by Wiggins, 1978. He used the varimax norm along the minimum entropy deconvolution in order to separate the components of a signal formed by the convolution of wavelet with the reflectivity function. The varimax norm has been used by other researchers to evaluate the quality of the deconvolution (Ooe \& Ulrych, 1979; Levi \& Oldenburg, 1982; Ulrych \& Walker, 1982). It measures the simplicity of a signal; the lower the number of wavelets distributed along the seismic trace, the greater the varimax norm value.

The mathematical equation used to calculate the varimax norm is given below.

$$
V(e)=\frac{\sum_{i=1} e_{i}^{4}}{\left(\sum_{i=1} e_{i}^{2}\right)^{2}} .
$$

For a single wavelet, the varimax norm gets its maximum value of 1 . If the result of the deconvolution is sparse/scattered and represented by many zeros, the trace is simple and varimax is has its greatest value. Figures 1a to $1 \mathrm{~d}$ show scattered signs with one, two, four and eight wavelets, respectively.

Figure 3 shows varimax norm values for the four signals displayed in Figure 2.

\section{PHASE SHIFT OPERATORS}

The frequency response of an all-pass filter equals 1 at all frequencies (Claerbout, 1985; Regalia et al., 1988; Reddy \& Swamy,
1988; Lang, 1998) where:

$$
\left|A\left(e^{i \omega}\right)\right|=1 .
$$

Equation (11) is valid for all $\omega$, the filter transfer function contains all poles and zeros in reciprocal conjugate pairs (Papoulis, 1977; Denbig, 1998; Oppenheim et al., 1996).

Considering $A(Z)$ as an all-pass filter of real coefficients,

$$
A(z)=\frac{Z^{-M} D\left(Z^{-1}\right)}{D(Z)} .
$$

The polynomial numerator is obtained from the denominator by reversing the order of the coefficients, as an example:

$$
A(z)=\frac{a_{2}+a_{1} Z^{-1}+1 Z^{-2}}{1+a_{1} Z^{-1}+a_{2} Z^{-2}} .
$$

\section{All-pass operator using the roots of the WL filter}

Consider $\tilde{C}(Z)$ the $Z$ transform (Oppenheim et al., 1996) of the Wiener-Levinson filter. The polynomial $\tilde{C}(Z)$ has all its roots with magnitude greater than one or, in the complex plane all roots are located outside the unit radius circle (Fig. 4). Considering order $M$, it is possible to divide the $M$ roots into two subsets of $a$ and $\beta$ such that $\alpha+\beta=M$. We can thus express as the product of these two polynomials,

$$
\tilde{C}(Z)={ }^{\alpha} F(Z){ }^{\beta} G(Z)
$$

where,

$$
\begin{aligned}
& { }^{\alpha} F(Z)=1+f_{1} Z+\cdots+f_{M-\beta} Z^{M-\beta} \\
& { }^{\beta} G(Z)=1+g_{1} Z+\cdots+g_{\beta} Z^{\beta}
\end{aligned}
$$

and, since $\tilde{C}(Z)$ is minimum phase, the ${ }^{\alpha} F(Z)$ and ${ }^{\beta} G(Z)$ are also minimum phase.

\section{Obtaining the Mixed-Phase Filter}

Using equations (15), it is possible to write the polynomial for the mixed-phase filter as follows:

$$
C(Z)={ }^{\alpha} F(Z)^{\beta} G\left(Z^{-1}\right) Z^{\beta} .
$$

Note that the term $\beta$ represents the maximum phase causal polynomial.

$$
{ }^{\beta} G\left(Z^{-1}\right) Z^{\beta}=g_{\beta}+\cdots+g_{1} Z^{\beta-1}+1 Z^{\beta} .
$$

Using the equations (14) and (16) we can write the equation for the polynomials associated with minimum and mixed-phase filters,

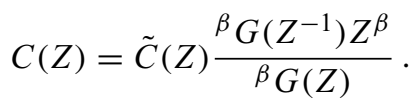




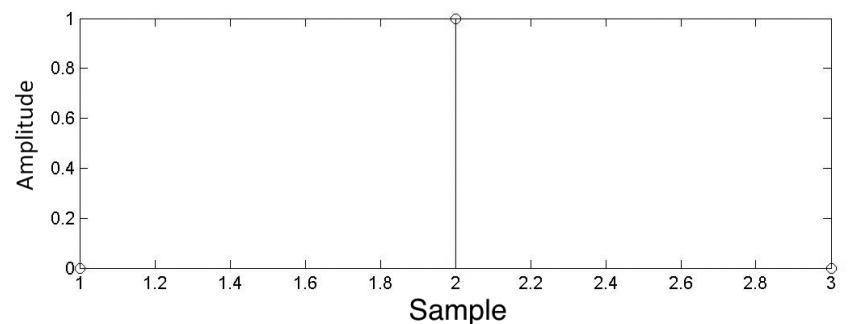

(a)

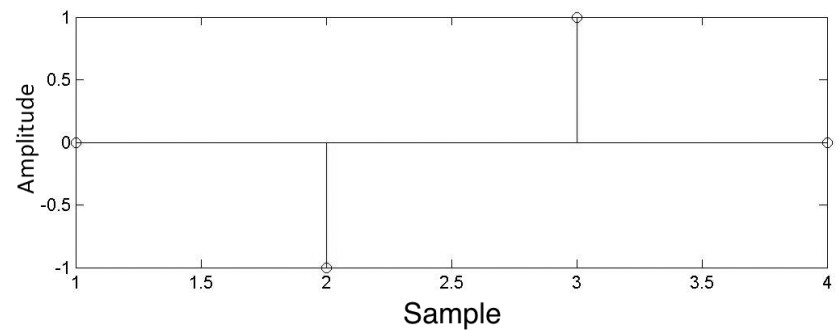

(b)

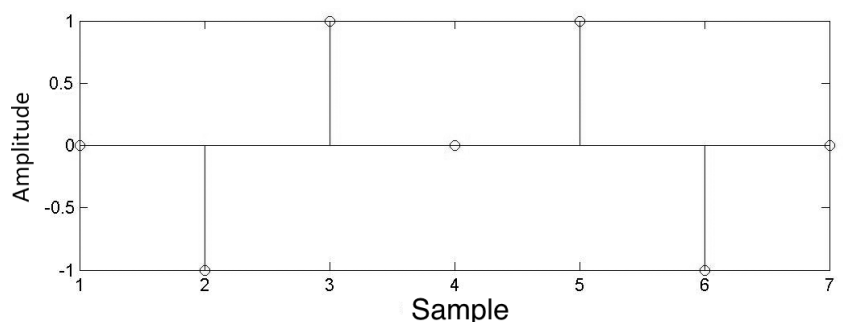

(c)

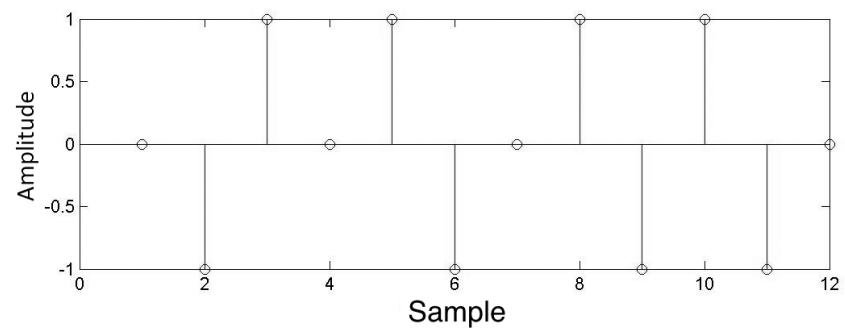

(d)

Figure 2 - Examples of discrete signals used to calculate the varimax norm. A single wavelet in (a) and two, four and eight wavelets for (b), (c) and (d), respectively.

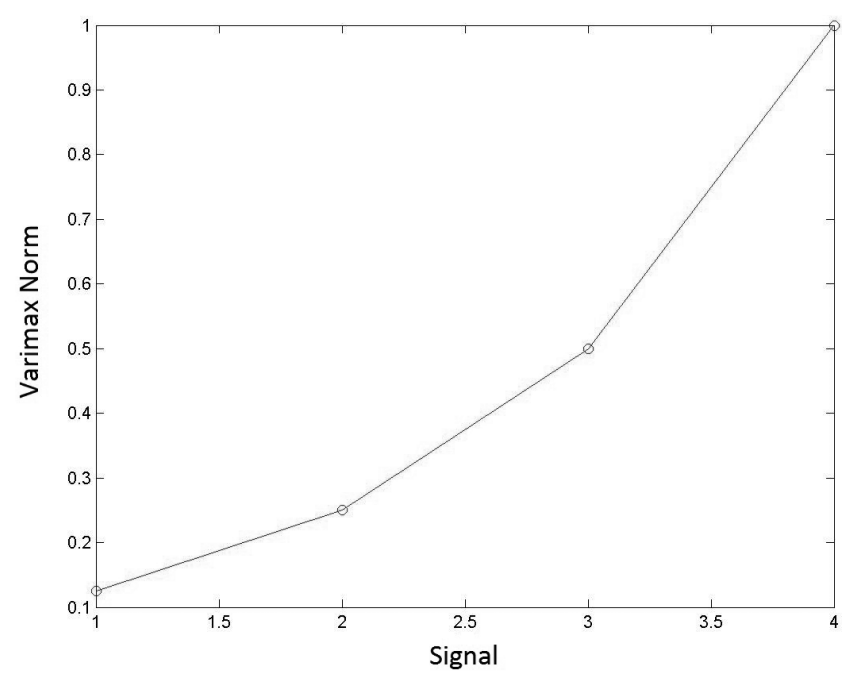

Figure 3 - Varimax norm values versus the signals represented in Figure 2. 


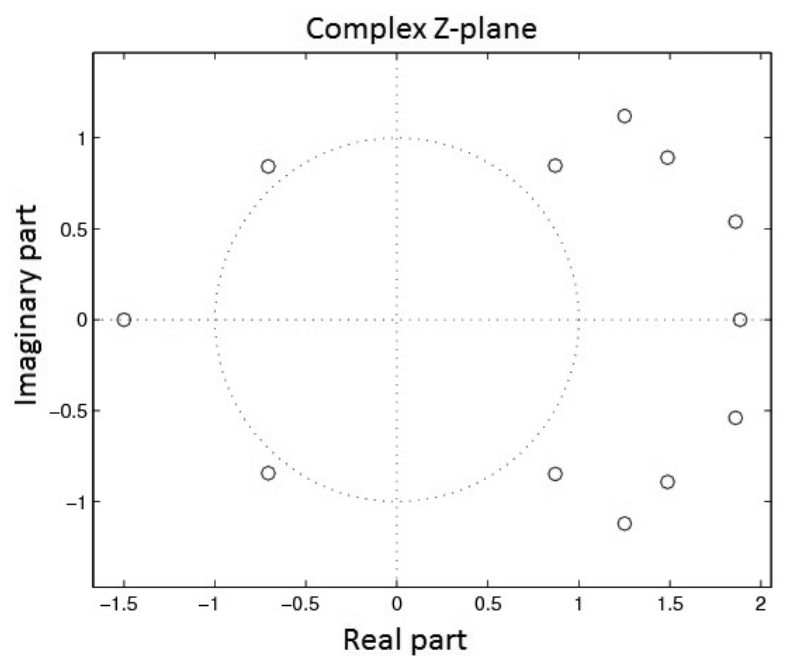

Figure 4 -Diagram in the complex $Z$-plane showing the roots of a minimum phase polynomial $C(Z)$.

We notice that the above equation yields the mixed-phase filter from the WL filter and a subset of roots estimated from the WL filter. Thus we have $\beta$ roots inside the unit radius circle, as illustrated in Figure 5.

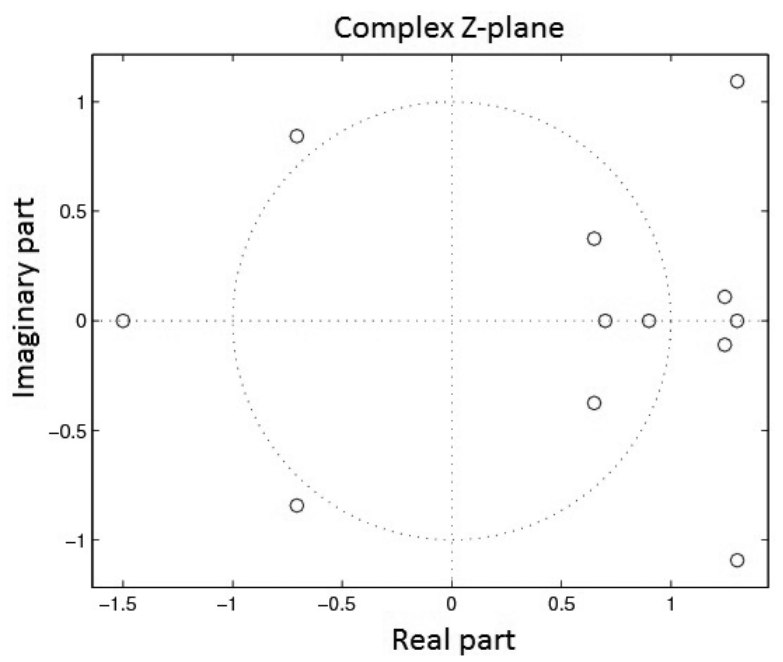

Figure $\mathbf{5}$-Diagram in the complex $Z$-plane showing the roots of a mixed-phase polynomial $C(Z)$.

\section{Obtaining the Mixed-Phase Wavelet}

The inverse of equation (18) yields the expression for calculating mixed-phase wavelet from the WL filter and some roots of the WL filter.

$$
P(Z)=\tilde{P}(Z) \frac{{ }^{\beta} G(Z)}{\beta G\left(Z^{-1}\right) Z^{\beta}} .
$$

With the filter WL $\tilde{C}(Z)$ and a $\beta$ subset of its roots, equations (18) and (19) can be used to get the inverse filter and the mixed-phase wavelet related to it.
Equations (18) and (19) are particularly important because the all-pass operator can be generated with roots calculated from the WL filter, which can be obtained simply for a finite number of coefficients (see Eq. (7)). For purposes of practical implementation of the algorithm we: (i) calculate the WL filter, (ii) calculate its roots and (iii) modify its phase through all-pass operator as shown in equation (18).

\section{Solving the optimization problem}

Considering, for example, that we have a total of 25 roots ( $M=$ 25 ) available for generating mixed-phase filters, of which 10 pairs are conjugate complex roots and 5 real roots, the total mixedphase filters that can be generated is equal to $2^{15}=32,768$. To determine the optimal filter from a universe of tens of thousands of possibilities implies a considerably high computational cost, since to evaluate filter performance, it is necessary to convolve with the panel of seismic traces under study. It is therefore desirable to have an optimized method for efficient selection of the optimum inverse filter.

Equation (18) yields mixed-phase filters from estimated or arbitrated roots of the minimum phase wavelet or WL inverse filter.

In the optimization problem proposed, the inverse filters $C(Z)$ are built from all-pass operators. The all-pass operators are responsible for reversing the subset of available roots into the unit circle. The binary condition of a particular root to be either outside or inside the unit circle makes the optimization problem suitable to be solved using a Genetic Algorithm (GA).

The optimization problem can be formulated as follows:

- A total of $\alpha$ roots is available (real or complex conjugate pairs of roots) greater than 1 , that is, complex roots outside the unit circle.

- $\beta$ subsets roots are extracted and used in equation (18) to create the all-pass operator that is used in the WL filter phase shift.

- The mixed-phase filters are generated and applied to the seismic data by convolution.

- Evaluation of filter performance is measured by the varimax norm.

- The GA described in the next section is used to optimize the process of choosing the optimal filter.

- If desired, at the end of the process, once the optimal inverse filter is known, its inverse can be used in equation (19) to estimate the seismic wavelet. 


\section{THE GENETIC ALGORITHM}

Genetic Algorithms (GAs) first pioneered by John Holland in 1975 were published in his book "Adaptation in Natural and Artificial Systems" as a method for optimizing nonlinear systems (Holland, 1975). The GAs basic concept is designed to simulate processes necessary for evolution, specifically those that follow the principles first laid down by Charles Darwin evolution theory and integrate the concepts of genetics and mechanisms that govern the reproduction of living organisms in a given population.

The GAs work with a population of individuals (models) in order to generate new individuals with improved characteristics over the generations. In the proposed optimization problem, individuals are the filters, which represent the inverse of the wavelet you want to collapse or reverse/invert.

The number of individuals in the population is chosen depending on the difficulty of the problem to be solved. If the number of individuals is small, the search universe may be insufficient and non-representative. On the other hand, with a very large number of individuals the required computing time can become unfeasible. Three types of coding are used most frequently: binary, Gray and real encoding.

The parameters are typically encoded in binary strings, using sets of numbers 0 and 1 to represent the variables (Goldberg, 1989). The Gray encoding uses binary encoding but it keeps a small perturbation rate in the parameters. Last, the real encoding works directly with real numbers; however, it makes genetic information exchange methods more complex.

The all-pass operators are responsible for reversing into the unit circle the subset of roots arbitrated or estimated by the WL filter. The binary state of a particular root being either inside or outside the unit circle can be conveniently represented by a binary string. Thus each all-pass operator, and consequently, each inverse filter, is associated with a binary string.

With the real roots, $\left\{z_{r, j}\right\}, j=1, \ldots, \omega$ and the complex conjugate pairs of roots, $\left\{z_{c, j}\right\}, j=1, \ldots, \gamma$ the polynomials of the first and second degree of real coefficients can be obtained,

$$
\begin{gathered}
{ }^{\omega} B_{j}(Z)=1-\frac{1}{z_{r, j}} Z, \quad j=1, \ldots, \omega, \\
{ }^{\gamma} B_{j}(Z)=\left(1-\frac{1}{z_{c, j}} Z\right)\left(1-\frac{1}{z_{c, j}^{*}} Z\right), \\
j=1, \ldots, \gamma .
\end{gathered}
$$

Each polynomial, ${ }^{\omega} B_{j}(Z)$ or $\gamma_{B_{j}}(Z)$, can be associated with one byte of a binary string, $J_{i}$, of random bytes as shown in Table 1.
Table 1 - Binary table showing the population of individuals (filters) used to create the all-pass operators.

\begin{tabular}{|c|c|c|c|}
\hline & ${ }^{\omega} B_{1}(Z)$ & $\ldots$ & ${ }^{\omega} B_{\omega}(Z)$ \\
\hline$J_{1}$ & 0 & $\ldots$ & 1 \\
$J_{2}$ & 1 & $\ldots$ & 0 \\
$\vdots$ & $\vdots$ & $\ldots$ & $\vdots$ \\
$J_{N \text { pop }}$ & 1 & $\ldots$ & 1 \\
\hline & $\gamma B_{1}(Z)$ & $\ldots$ & $\gamma B_{\gamma}(Z)$ \\
\hline$J_{1}$ & 1 & $\ldots$ & 0 \\
$J_{2}$ & 1 & $\ldots$ & 0 \\
$\vdots$ & $\vdots$ & $\ldots$ & $\vdots$ \\
$J_{N \text { pop }}$ & 0 & $\ldots$ & 1 \\
\hline
\end{tabular}

The minimum phase polynomials ${ }^{\beta} B_{i}(Z)$ used for the inverse filter are obtained from the product of the polynomials associated with non-zero bytes, along each row of Table 1 ,

$$
{ }^{\beta} B_{i}(Z)=\prod_{j=1}^{\omega}{ }^{\omega} B_{j}(Z) J_{j} \prod_{j=1}^{\gamma}{ }^{\gamma} B_{j}(Z) J_{j}
$$

where $J_{j}$ is equal to zero or 1 .

\section{Application of genetic operators}

The genetic operators are necessary to diversify the population maintaining the adaptation traits acquired by previous generations. In most cases, GAs use three operators: selection, crossover and mutation.

\section{Selection}

This genetic operator, also called reproduction, selects the individuals that will undergo crossover and mutation. Similar to the process of natural selection, the fittest individuals are more likely to be chosen, according to merit assessment (varimax norm).

\section{Crossover}

The goal of crossover is to permute genetic material between pairs of previously selected individuals. After the pairs are formed, the individuals undergo the crosslinking process, which may or may not occur, according to a given crossover probability. This genetic operator is largely responsible for creating new individuals; similar to what happens in nature, where most couples have children.

The GAs are characterized by high implementation flexibility, which also applies to the crossover process that can be done in different ways. Table 2 shows the crossover with chromosomes permutation. 
Table 2 - Crossover process with permutations of genetic content.

\begin{tabular}{|l|l|l|l|l|l|}
\hline parents & 11001 & crossover & $110\langle 01\rangle$ & children & 11011 \\
parents & 01111 & crossover & $011\langle 11\rangle$ & children & 01101 \\
\hline
\end{tabular}

\section{Mutation}

Mutation prevents premature convergence. Mutation is understood as the insertion of new genetic material in the population. This process, just as the crossover, may or may not occur according to a given probability of mutation (pmut). This probability should be very low, from 0 to $5 \%$, so that the pursuit of the optimum individual is not purely random. This is analogous to nature behavior, where mutations and abnormalities are rarely seen. As in crossover, mutation may be accomplished in many ways, one of which is shown in Table 3.

Table 3 - Mutation process showing the random exchange of one byte of the binary string associated with the model parameter.

\begin{tabular}{|l|l|l|l|}
\hline mutation & $1\langle 1\rangle 001$ & son & 10011 \\
\hline
\end{tabular}

Figure 6 shows the GA flowchart and the performance of the three genetic operators.

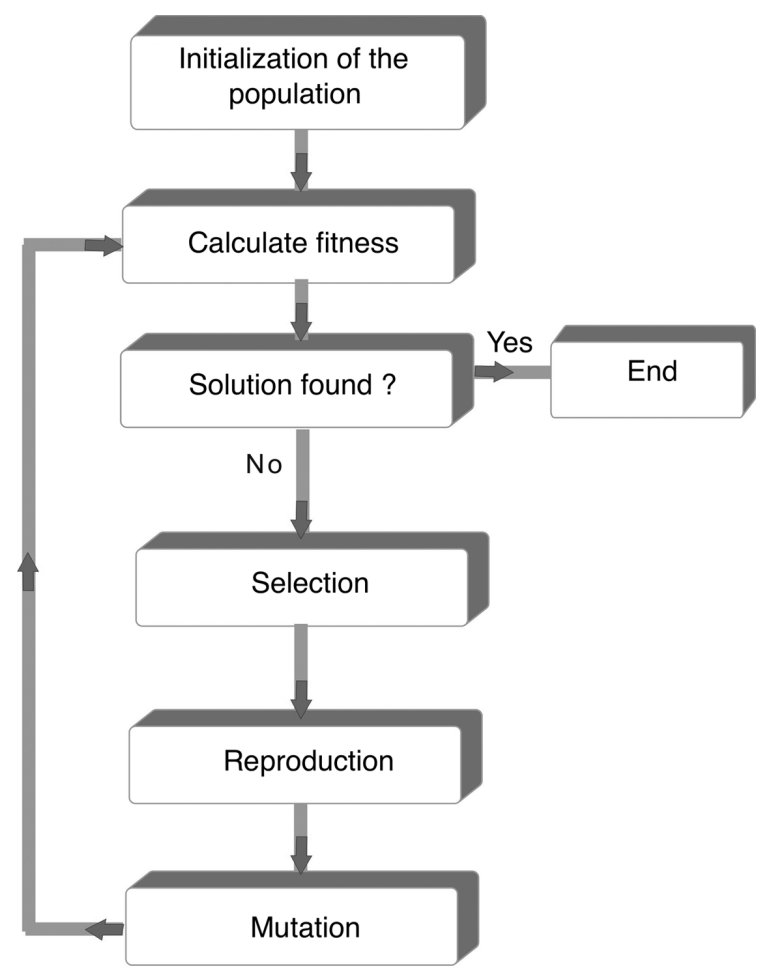

Figure 6 - Flowchart of the Genetic Algorithm (GA) showing how genetic operators generate new populations.

\section{RESULTS USING THE ALL-PASS OPERATOR FROM THE WIENER-LEVINSON FILTER}

The WL filter has all roots necessary to deconvolve the minimum phase wavelet and any other that has the same FAC. This approach is considerably simpler than that described by Porsani \& Ursin, (2000) and Ursin \& Porsani (2000). The approach proposed in this work is simpler because it uses directly the WL filter of $N$ coefficients. The examples presented in this paper use $N=25$. Obtaining the WL filter and its roots is fairly simple.

The procedure consisted of the following steps:

- The WL inverse filter of 25 coefficients and its minimum phase wavelet were calculated.

- Equation (18) was used to obtain the all-pass operators.

- The GA was used to obtain the optimal inverse filter. The parameters used were: number of generations $N g=30$, the number of models (strings) $\mathrm{Nm}=50$ and mutation probability $p_{m}=0.2$.

- The trace(s) of the desired seismic section were/was deconvolved.

- Equation (19) was used to estimate the seismic wavelet.

To test the new approach, the algorithm was initially applied on synthetic data to deconvolve the mixed-phase wavelet, followed by synthetic seismic trace and finally real seismic data. The results were compared with those obtained with the conventional WL deconvolution method.

\section{Deconvolution and estimation of wavelet}

Figures 7 and 8 show the result of the mixed-phase wavelet deconvolution.

Figure 7a shows the synthetic mixed-phase wavelet used. Figures $7 \mathrm{~b}$ and $7 \mathrm{c}$ show the estimated minimum and mixedphase wavelets and Figure $7 \mathrm{~d}$ shows the optimal mixed-phase filter. There is good similarity between the estimated mixed-phase wavelet and the original wavelet of Figure 7a.

Figure 8 shows the varimax norm values calculated for over 30 generations of the GA. The three curves represent the maximum, average and minimum values for the 50-model population. It is noteworthy that the average convergence curve quickly approaches the maximum curve, indicating that over generations the population is becoming more and more homogeneous around the best model (optimal filter). 


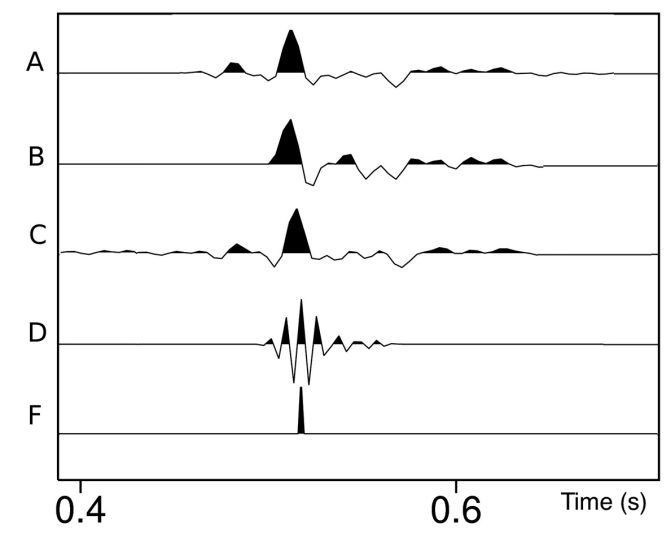

Figure 7 - Wavelet deconvolution and estimation. The mixed-phase wavelet in (A), minimum and mixed-phase wavelets in (B) and (C), the optimal mixed-phase filter in (D), and the result of the original wavelet deconvolution with the inverse filter estimated in (E).

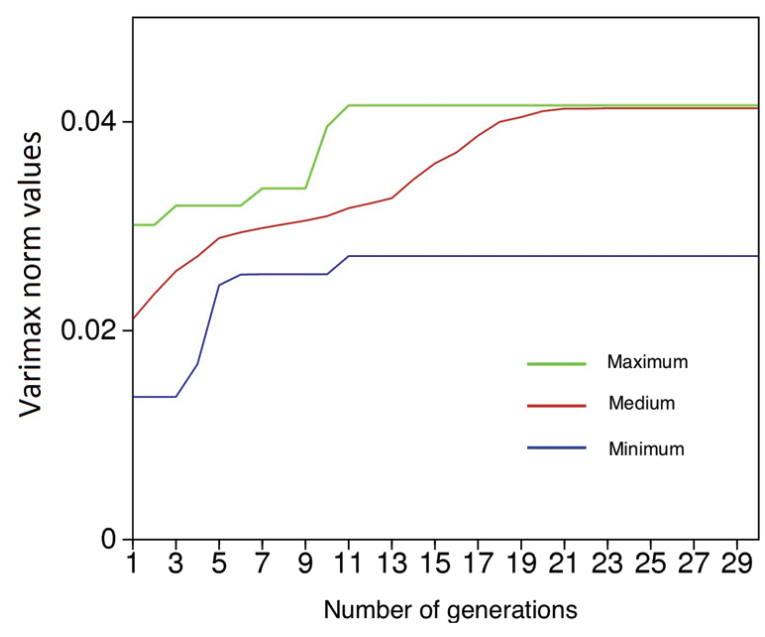

Figure 8 - GA performance in synthetic wavelet deconvolution shown in Figure 7A. Maximum, medium and minimum varimax norm values of the population over 30 generations. Ascending curves illustrate GA convergence behavior.

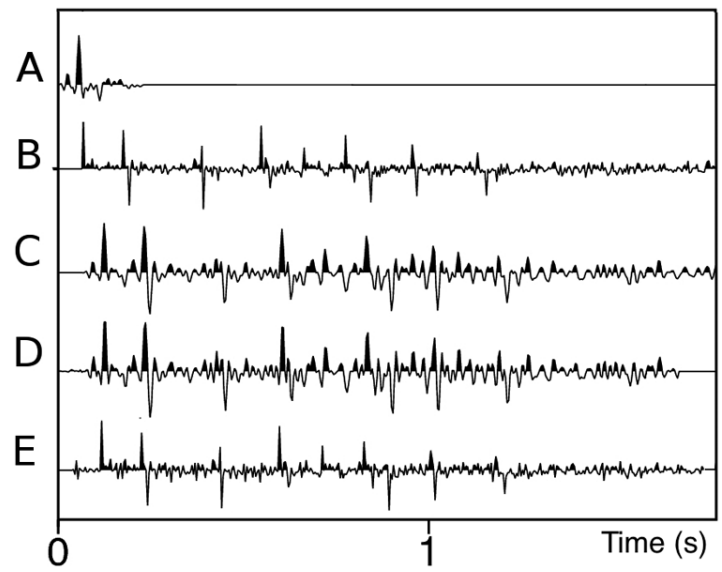

Figure 9 - Synthetic trace deconvolution. Mixed-phase wavelet (A), pseudorandom reflectivity function (B) and synthetic seismic trace (C). Deconvolution of minimum (D) and mixed-phases with optimal filter (E).
Figures $10 \mathrm{~A}$ and $10 \mathrm{~B}$ show the estimated minimum and mixed-phase wavelet, respectively. Good similarity is observed between the estimated mixed-phase wavelet and the original wavelet (Fig. 10A) used to generate the synthetic trace. Figure 10C shows the optimal inverse filter obtained with GA.

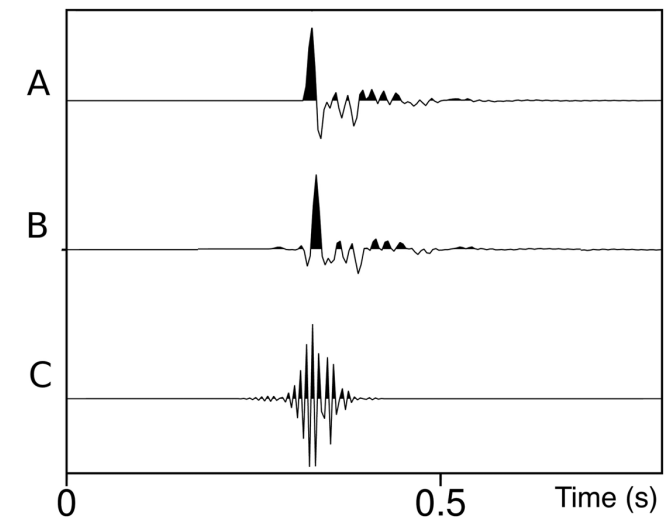

Figure 10 - Wavelet and optimal filter estimates. Minimum phase wavelet (A). Mixed-phase wavelet (B) and its corresponding optimal inverse filter (C).

Figure 11 shows the GA performance to obtain the optimal filter that deconvolves the synthetic trace of Figure 10. The figure illustrates the evolution of the maximum, average and minimum varimax norm values, calculated at the end of each of the $30 \mathrm{GA}$ generations. The same converging behavior towards increasing varimax values is observed for all three curves.

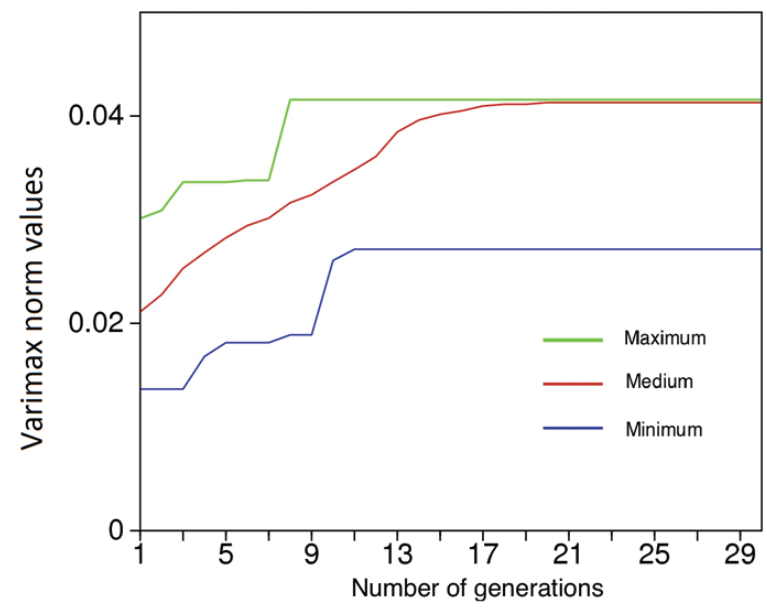

Figure 11 - GA performance in the estimation of the optimal mixed-phase filter that deconvolves the synthetic trace of Figure 10C.

\section{Deconvolution of 2D seismic sections}

Figure 12 shows a post-stack seismic marine section of Jequitinhonha Basin. A single filter is calculated and applied in the deconvolution of the entire seismic section, to do so the following procedure was followed: 


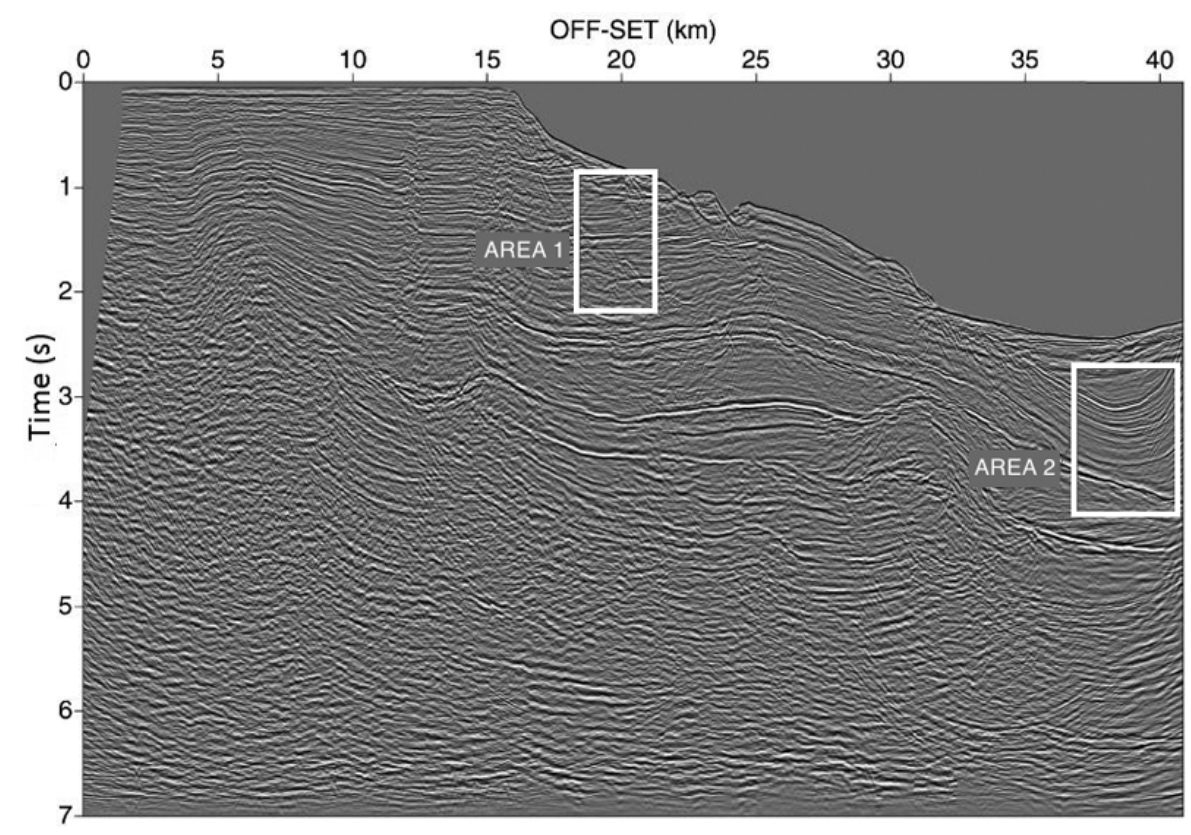

Figure 12 - Post-stack marine seismic section of the Jequitinhonha Basin showing areas 1 and 2 chosen to illustrate the method.

- The window of interest where the varimax norm will be evaluated is defined.

- The mean autocorrelations for the traces within the window are calculated.

- The WL filter of $N=25$ coefficients is calculated.

- The optimal inverse filter is calculated using the GA and the section is deconvolved.

- The wavelet is estimated by the inverse of the optimal filter.

Figure 12 shows the selected areas 1 and 2 chosen to illustrate the method. Figures $13 \mathrm{~A}$ and $14 \mathrm{~A}$ show the original data in greater detail. Figures 13B and 14B show the minimum phase WL deconvolution results. Figures $13 \mathrm{C}$ and $14 \mathrm{C}$ show the mixed-phase deconvolution results using the optimum filter obtained with the GA.

The mixed-phase deconvolution results show some improvement in the temporal resolution and the lateral continuity of reflections, compared to the results obtained with the classical minimum phase deconvolution method or WL method.

Figure 15 shows the average amplitude spectrum of original and deconvolved seismic sections. It should be noted that the all-pass operator modified only the WL phase filter, leaving the spectral amplitude similar to the result of the mixed-phase filter deconvolution.

\section{Deconvolution results on 3-D seismic volume}

The method was applied to the 3-D seismic section available in the OpendTect software, in the Netherlands Offshore F3 Block. Figure 16 shows the seismic section used in the test method.

Figure 17 shows the results of the mixed and minimum phase deconvolution on a real seismic trace, extracted from Figure 16 and shown in Figure 17A. Figures 17B and $17 \mathrm{C}$ show the results of the WL and mixed-phase deconvolution using the optimal filter. It is noteworthy the improved definition of the reflections obtained with the mixed-phase deconvolution approach.

Figures $18 \mathrm{~A}, 18 \mathrm{~B}$ and $18 \mathrm{C}$ show, respectively, the estimated minimum and mixed-phase wavelets and, the inverse mixedphase filter used to deconvolve the trace in Figure 18A.

Figure 19 shows the result of deconvolution on a panel of traces extracted from the 3D volume (Fig. 16). The deconvolved seismic section is shown using the shaded relief feature (Barnes, 2003) of the SU (Seismic Unix) software. Some improvement in the resolution of the individualized thin layers, most visible in the lower left section, can be observed.

\section{CONCLUSIONS}

We present a new approach to the deconvolution of a wavelet. The method uses the Wiener-Levinson filter to estimate the wavelet related roots that should be inverted. The roots of the polynomial associated with the WL filter allow generating a set of possible mixed-phase filter that can be implemented and tested. These 


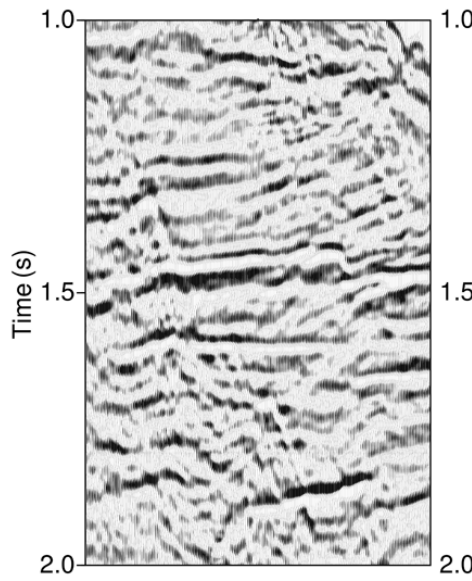

(A)

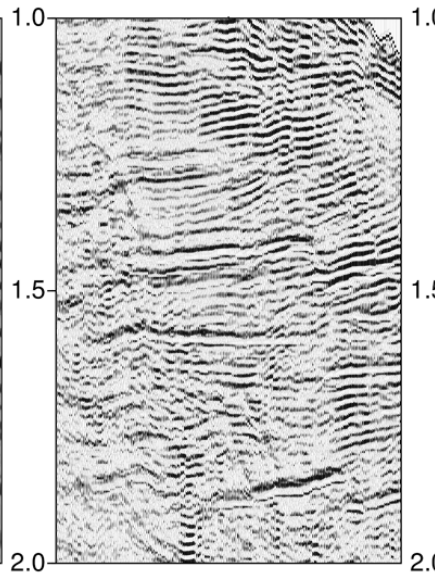

(B)

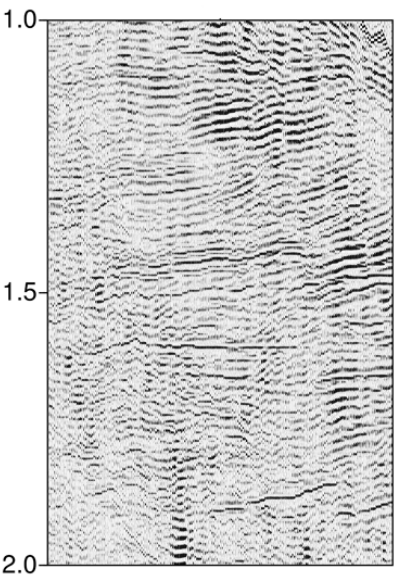

(C)

Figure 13 - Area 1 deconvolution results. Original section (A), WL deconvolution result (B) and optimal mixed-phase filter deconvolution (C).

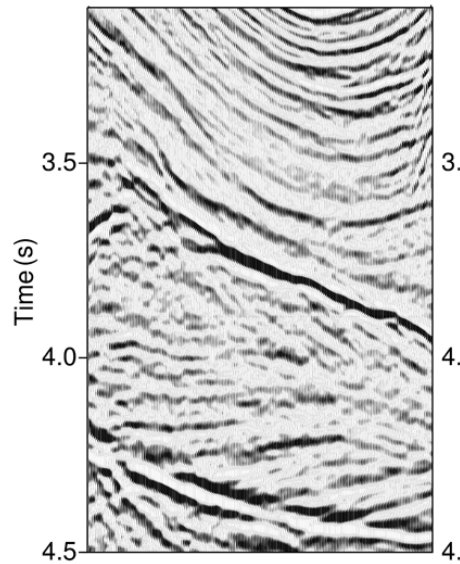

(A)

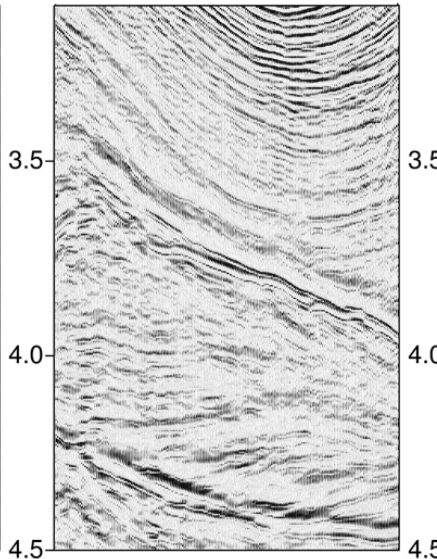

(B)

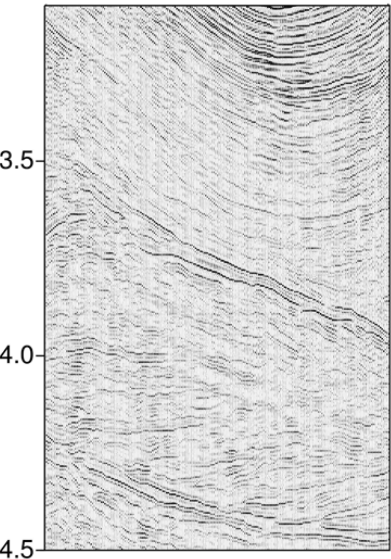

(C)

Figure 14 - Area 2 deconvolution results. Original section (A), WL deconvolution result (B) and optimal mixed-phase filter deconvolution (C).

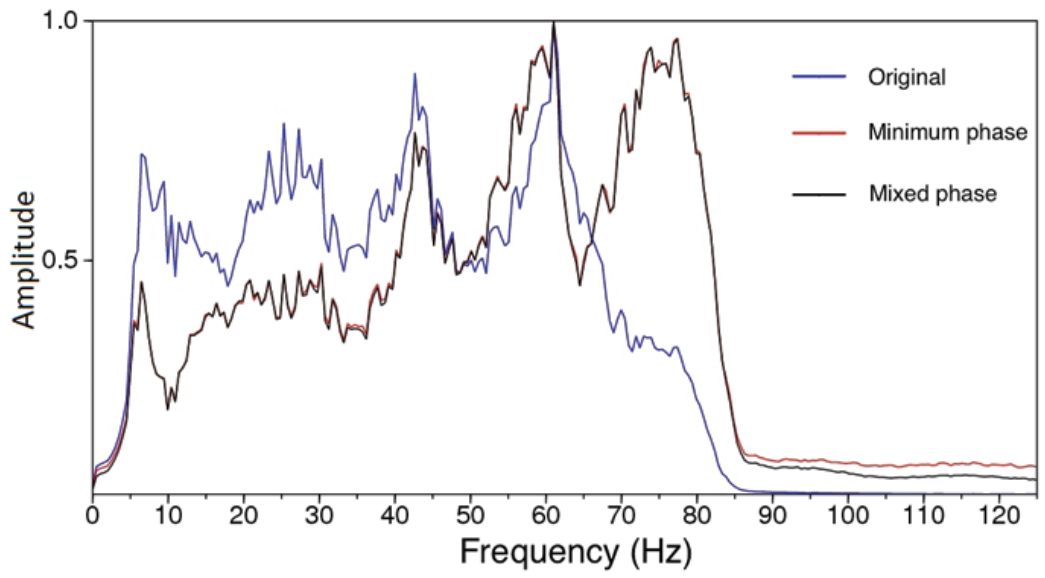

Figure 15 - Average amplitude spectra of the original and deconvolved data for Area 1. 


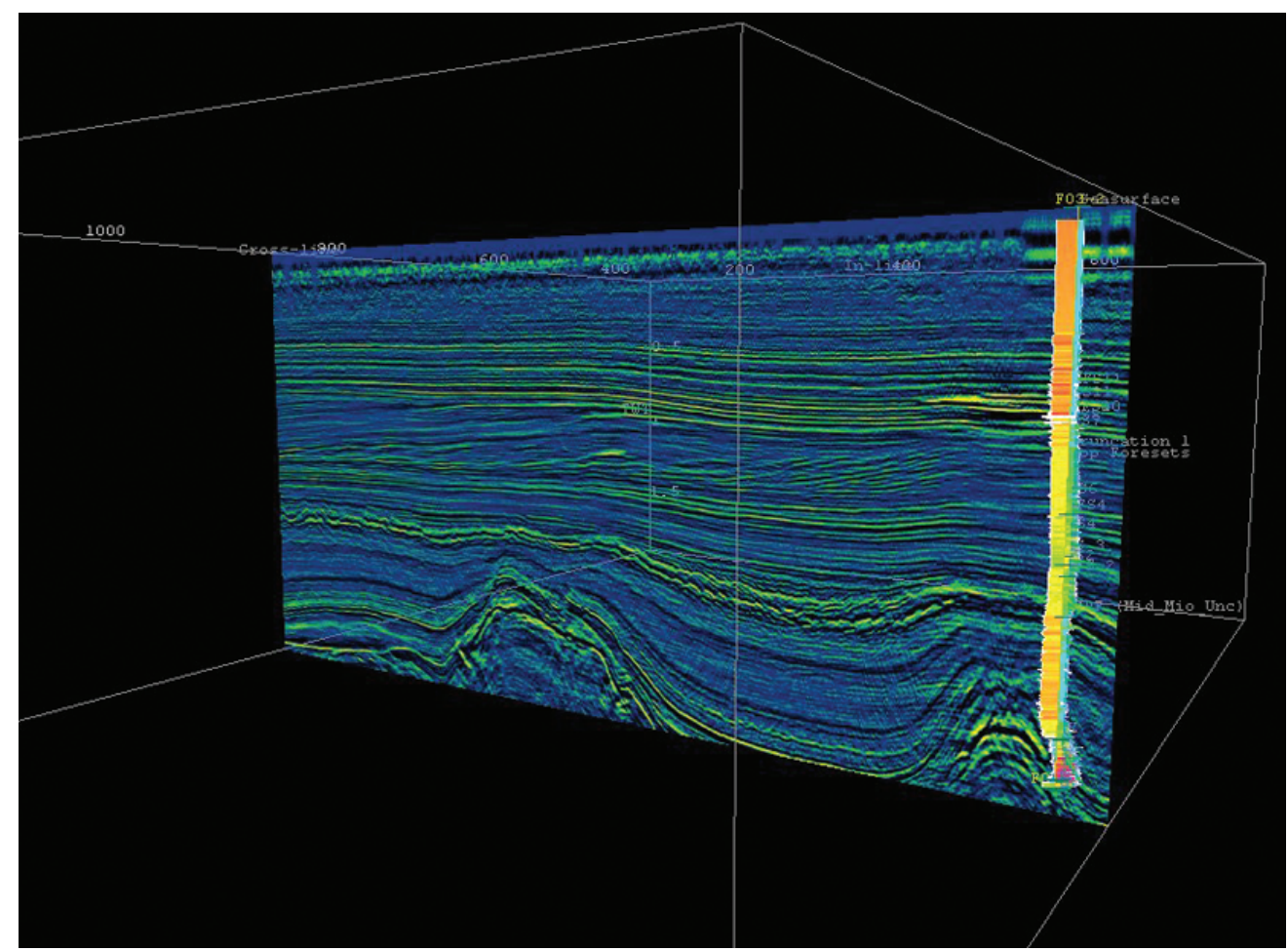

Figure 16 - 3-D seismic data/section, Netherlands Offshore F3 Block, using OpendTect application.

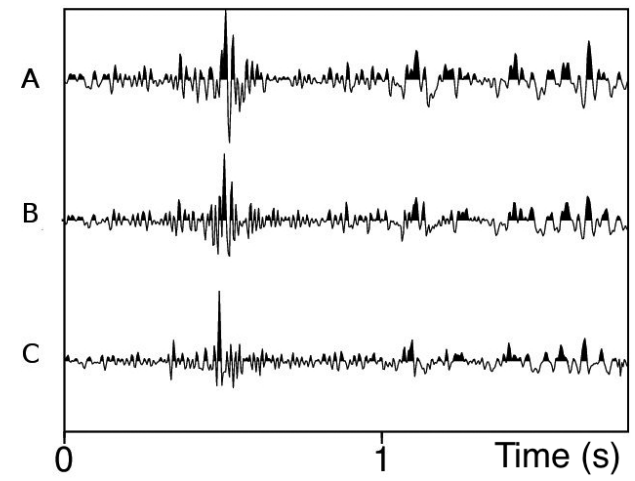

Figure 17 - Original seismic trace (A), WL deconvolution (B) and optimal mixed-phase filter deconvolution (C).

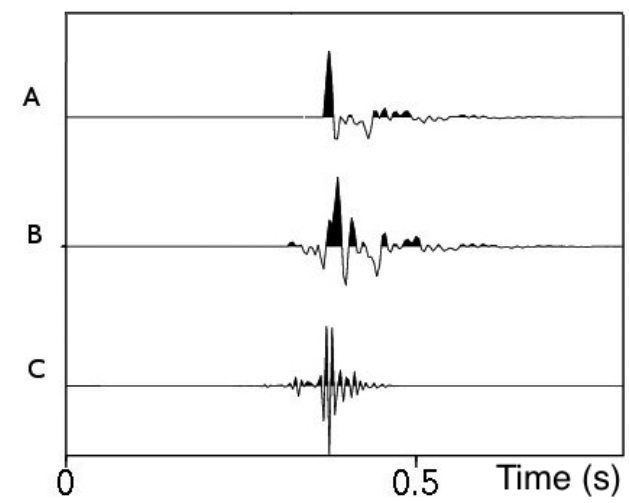

Figure 18 - Minimum and mixed-phase wavelets (A) and (B), respectively, and optimal mixed-phase filter (C). 


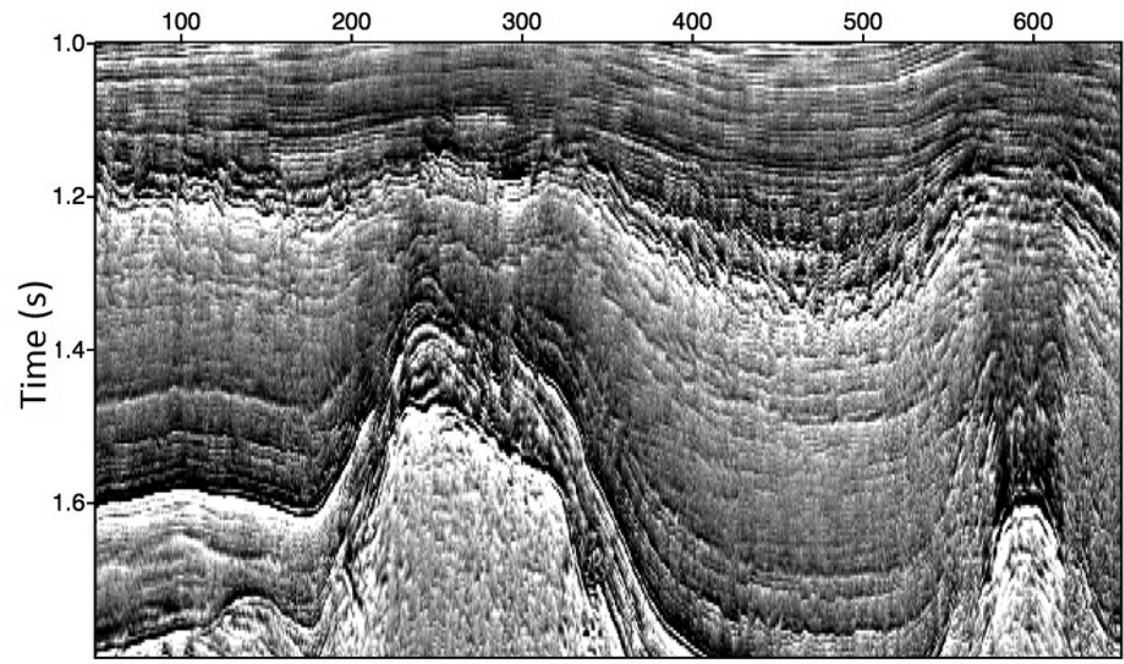

(A)

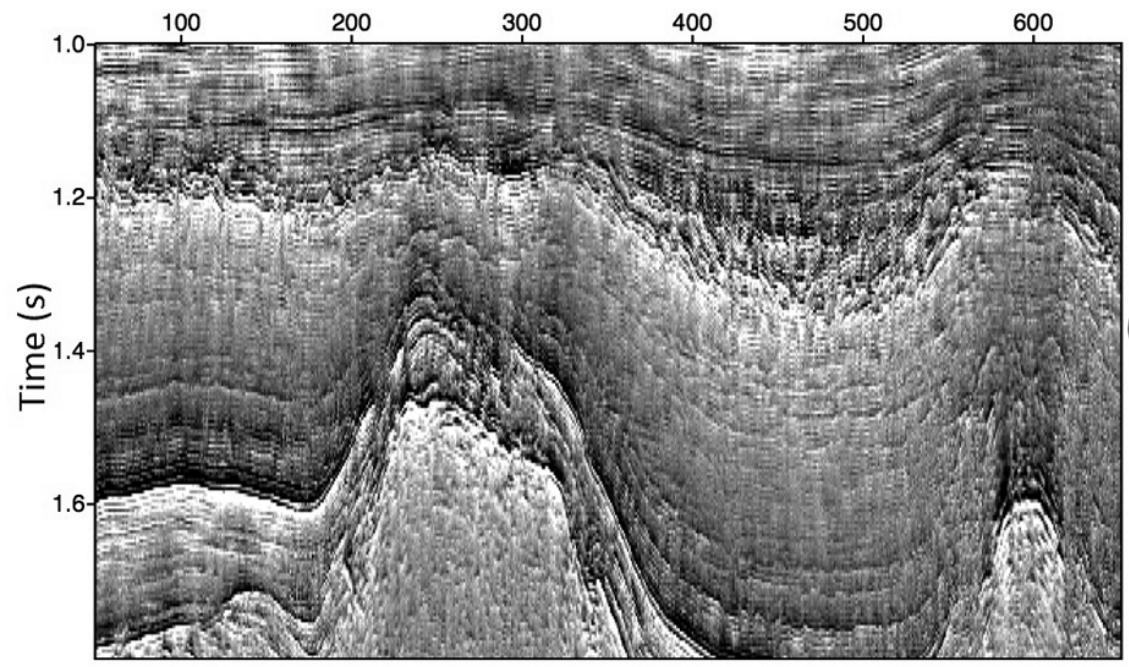

(B)

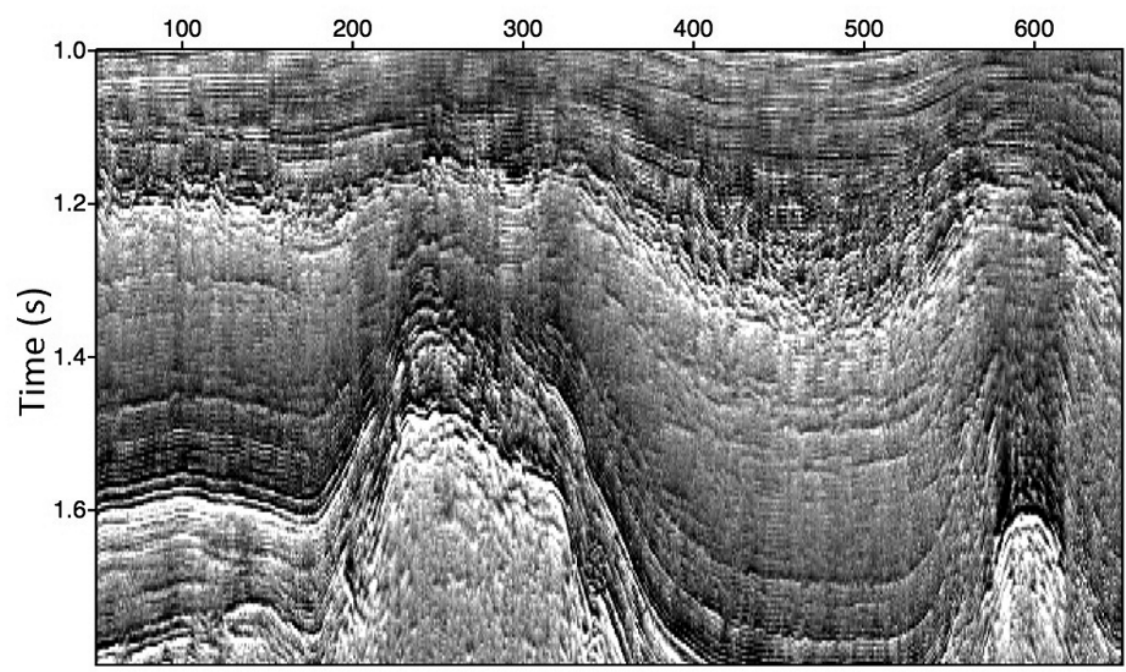

(C)

Figure 19 - Seismic section with reservoir zone. Original section (A), WL deconvolution (B) and mixed-phase deconvolution (C). 
filters are generated through all-pass operators that modify only the filter phase and a genetic algorithm is used to choose the optimal filter. Once the best filter is chosen, the seismic wavelet is obtained. The optimal mixed-phase filters were tested on synthetic and real data showing the effectiveness of the new approach when the phase characteristics of the wavelet are not known.

The deconvolution method using all-pass operators is less computationally efficient than the conventional WL method; however, this disadvantage is compensated with the use of multiprocessors. The results indicate that the new approach for deconvolution of seismic data can be used to improve the identification of reflected seismic events and show in greater detail the earthquake-stratigraphic and structural features of interest to the exploration and study of hydrocarbon reservoirs.

\section{REFERENCES}

BARNES AB. 2003. Shaded relief seismic attribute. Geophysics, 68: 1281-1285

CLAERBOUT J. 1985. Fundamentals of geophysical data processing. Blackwell Science Inc., 274 pp.

DENBIG P. 1998. System analysis and signal processing with emphasis on the use of MATLAB. Addison-Wesley, 513 pp.

EISNER A \& HAMPSON G. 1990. Decomposition into minimum and maximum-phase components. Geophysics, 55: 897-901.

GOLDBERG DE. 1989. Genetic algorithms in search, optimization, and machine learning. Reading, MA. Addison-Wesley, 432 pp.

HOLLAND JH. 1975. Adaptation in natural and artificial systems. University of Michigan, Press, USA, 183 pp.

LANG M. 1998. All-pass filter design and applications. IEEE Transactions on Signal Processing, 46: 2505-2514.

LAZEAR GD. 1993. Mixed-phase wavelet estimation using fourth order cumulants. Geophysics, 58: 1042-1051.

LEINBACH J. 1993. Wiener spiking deconvolution and minimum phase wavelet: a tutorial. Leading Edge, 14: 189-192.

LEVI S \& OLDENBURG DW. 1982. The deconvolution of phase shifted wavelet. Geophysics, 47: 1285-1294.

LU X \& WANG Y. 2007. Mixed-phase wavelet estimation by iterative linear inversion of high-order statistics. Geophysics, 6: 21-28.

MISRA S \& CHOPRA S. 2010. Phase stability via nonlinear optimization: A case study. Leading Edge, 29: 1338-1343.

MISRA S \& SACCHI MD. 2006. Nonminimun phase wavelet estimation by nonlinear optimization of allpass operators. Geoph. Prosp., 55: 223234.
OOE M \& ULRYCH TJ. 1979. Minimum entropy deconvolution with an exponential transformation. Geoph. Prosp., 27: 458-473.

OPPENHEIM AV, WILLSKY AS \& NAWAB SH. 1996. Signals and systems, 2nd ed. Prentice Hall, Inc., 957 pp.

PAPOULIS A. 1977. Signal analysis. McGraw-Hill Companies, 431 pp.

PORSANI M \& URSIN B. 2000. Mixed-phase deconvolution and wavelet estimation. Leading Edge, 19: 76-79.

PORSANI MJ \& URSIN B. 1998. Mixed-phase deconvolution. Geophysics, 63: 637-647.

REDDY, GR \& SWAMY MNS. 1988. Digital all-pass filter design through discrete hilbert transform. Proceedings of the IEEE International Conference on Acoustics, Speech, and Signal Processing, 150-156.

REGALIA PA, MITRA SK \& VAIDYANATHAN PP. 1988. The digital allpass filter. A versatil signal processing building block. Proceedings of the IEEE, 76: 19-37.

ROBINSON EA. 1954. Predictive decomposition of time series with applications to seismic exploration. Ph.D. thesis, Geology and Geophysics, Massachusetts Institute of Technology. 255 pp.

ROBINSON EA. 1967. Predictive deconvolution of time series with application to seismic exploration. Geophysics, 32: 418-484.

ROBINSON EA \& TREITEL S. 1982. Geophysical signal analysis. Prentice-Hall, Inc., 466 pp.

SACCHI MD \& ULRYCH TJ. 2000. Non minimum-phase wavelet estimation using higher order statistics. Leading Edge, 19: 80-83.

ULRYCH TJ \& TREITEL S. 1991. A new proof of the minimum phase property of the unit delay prediction error operator. IEEE Transactions on Signal Processing, 39: 252-254.

ULRYCH TJ \& WALKER C. 1982. Analytic minimum entropy deconvolution. Geophysics, 36: 467-481.

URSIN B \& PORSANI MJ. 2000. Estimation of an optimal mixed-phase inverse filter. Geoph. Prosp., 19: 76-79.

VAN DER BAAN M. 2008. Time varing wavelet estimation and deconvolution by kutorsis maximization. Geophysics, 73: V11-V18.

VAN DER BAAN M \& PHAM DT. 2008. Robust wavelet estimation and blind deconvolution of noisy surface seismics. Geophysics, 73: V37V46.

VELIS DR \& ULRYCH T. 1996. Non minimun phase wavelet estimation using higher order statistics. Leading Edge, 61: 1939-1948.

WIGGINS RA. 1978. Minimun entropy deconvolution. Geoexploration, 16: 21-31.

YILMAZ 0. 1987. Seismic data processing. Society of Exploration Geophysicists, 526 pp. 


\section{NOTES ABOUT THE AUTHORS}

Dorian Caraballo Ledesma. Completed the undergraduate course in Physics at the Universidad Industrial de Santander, Bucaramanga, Colombia, in 2004. Participated in several signal processing projects when he acquired theoretical and practical knowledge in various fields of physics. Earned a PhD in Exploration Geophysics at the Universidade Federal da Bahia in 2011, with the Oil Exploration group, worked on deconvolution and wavelet estimation for improving the resolution of seismic imaging and reservoir characterization. Currently working as a consultant geophysicist at the Instituto Colombiano del Petróleo (ICP - Colombian Petroleum Institute) of Ecopetrol.

Milton José Porsani. Graduated in Geology from the Universidade de São Paulo (1976) and Education at the Universidade de São Paulo (1978). Holds a Master degree in Geophysics from the Universidade Federal do Pará (1981), PhD degree in Geophysics from the Universidade Federal da Bahia (1986) and a postdoctoral internship at the Institute of Geophysics of the University of Texas (September 1992 to March 1994). Professor in the Department of Geology and Applied Geophysics, UFBA since January 1990 and Chairman since 2000. Researcher of CNPq since 1990 and Researcher 1A level since 2004. He was coordinator of the Cooperative Research Network NNE (FINEP) from 2003 to June 2009. He is currently coordinator of the Instituto Nacional de Ciência e Tecnologia de Geofísica do Petróleo from CNPq (INCT-GP) and vice-coordinator of the Cooperative Research Network NNE (FINEP) in Geophysics Exploration. He is a member of the Câmara Técnica de Ciências Matemáticas e Naturais da FAPESB - Foundation for Research Support of the State of Bahia, since 2009 and a member of the Academy of Sciences of Bahia since 2011. His areas of interest include: the study of inverse problems in geophysics, seismic processing, development of methods and algorithms for filtering and inversion of seismic data, groundwater exploration and geophysical studies geared to monitor contamination plumes and environment quality control. 